Rev. Bras. Cir. Cardiovasc.

5(3): 149-153, 1990.

\title{
Cirurgia das dissecções crônicas da aorta ascendente com insuficiência valvar
}

\author{
Paulo M. PÊGO-FERNANDES*, Noedir A. G. STOLF*, Ronaldo D. FONTES*, Geraldo VERGINELLI*,
} Adib D. JATENE*

RBCCV 44205-118

PÊGO-FERNANDES, P. M.; STOLF, N. A. G.; FONTES, R. D.; VERGINELLI, G.; JATENE, A. D. - Cirurgia das dissecçōes crônicas da aorta ascendente com insuficiência valvar. Rev. Bras. Cir. Cardiovasc., 5(3): 149-153, 1990.

RESUMO: No período de janeiro de 1980 a dezembro de 1988, foram operados 44 pacientes com dissecçōes aórticas crônicas e insuficiência aórtica. Esse grupo foi analisado para se avaliar a evoluçāo comparativa dos doentes em que a valva aórtica foi preservada em relação àqueles em que houve substituiçāo valvar. As características pré-operatórias eram semelhantes, sendo efetuada troca da valva quando havia degeneração valvar ou ectasia ânulo-aórtica. Nos casos de desabamento de válvulas com alargamento do anel realizou-se plástica valvar. Em $48 \%$ dos casos foi possível a preservaçāo valvar através de suspensão da valva aórtica. Nos 23 doentes em que foi realizada a substituição valvar, a técnica de Bentall e De Bono foi utilizada em 16. Em seis pacientes foram associados outros procedimentos cirúrgicos. Em todos os doentes operados a partir de 1986 foi utilizada cola biológica. Em 41 (93\%) pacientes a aorta proximnal foi substituída e nos três restantes realizou-se aortoplastia. Cinco pacientes $(11 \%)$ tiveram morte hospitalar, três por baixo débito, um por sangramento e um por complicaçāo neurológica. Dois pacientes $(4 \%)$ apresentaram morte tardia. O seguimento dos 37 sobreviventes variou de dois a 108 meses, com média de $18: 78 \%$ estavam em classe l e os demais em classe II. Dois pacientes que tiveram a valva preservada apresentaram insuficiência aórtica discreta. Três doentes que receberam válvula biológica necessitaram reoperação tardiamente, por disfunção da válvula. Um doente submetido, inicialmente, a aortoplastia e plástica valvar apresentou redissecção e insuficiência aórtica após 60 meses, sendo reoperado pela técnica de Bentall. No estudo com curva atuarial de sobrevida notamos que os pacientes submetidos a plástica valvar tiveram maior sobrevida. Podemos concluir que: 1) a suspensāo valvar é uma técnica satisfatória em pacientes com dissecçōes crônicas da aorta, com baixa mortalidade e menor índice de complicaçōes a médio prazo do que a substituiçāo valvar; 2) a identificaçāo do mecanismno da insuficiência valvar é fundamental para decisāo da tática operatória; 3) - uso da cola biológica facilita o manuseio da aorta e pode diminuir o sangramento intra-operatório; 4) quando é necessária a substituição valvar tem-se preferido empregar prótese mecânica dada a maior dificuldade técnica na reoperação nesses pacientes; 5) a aortoplastia não deve ser utilizada devido à alta incidência de redisseç̧äo aórtica.

DESCRITORES: aorta, dissecção, cirurgia; valva aórtica, cirurgia.

\section{INTRODUÇĀO}

Nas dissecçōes agudas da aorta proximal em que exista insuficiência valvar, há praticamente unanimidade na tendência à preservação da valva aórtica, desde que não exista acometimento primário dessa valva ${ }^{14,17,19}$. Já, nas dissecçōes crônicas com insuficiência aórtica, muitos realizaram a substituiçāo valvar com grande freqüência ${ }^{9}$ 10, 14, 21,23 . No Instituto do Coração, no inicio da experiência ${ }^{23}$, também substituíamos a valva com maior freqüência, enquanto que atualmente procura-se preservar o aparelho valvar.

O objetivo deste trabalho é a análise da evoluçāo dos pacientes portadores de dissecção aórtica

Trabalho realizado no Instituto do Coração do Hospital das Clínicas da Faculdade de Medicina da Universidade de Sâo Paulo. São Paulo, SP. Brasil. Apresentado ao $17^{\circ}$ Congresso Nacional de Cirurgia Cardíaca. Belo Horizonte. MG, 6 e 7 de abril, 1990.

* Do Instituto do Coraçáo do Hospital das Clínicas da Faculdade de Medicina da Universidade de Sâo Paulo.

Endereço para separatas: Paulo Pêgo-Fernandes. Av. Dr. Eneas de Carvalho Aguiar. 44. Divisão Cirúrgica. 05403 São Paulo, SP. Brasil. 
PÊGo-FERnANDES, P. M.; STOLF, N. A. G.; FONTES, R. D.; VERGINELLI, G.; JATENE, A. D. - Cirurgia das dissecçōes crônicas da aorta ascendente com insuficiência valvar. Rev. Bras. Cir. Cardiovasc., 5(3): 149-153, 1990.

crônica com insuficiência aórtica, que foram operados no Instituto do Coração.

\section{CASUÍSTICA E MÉTODOS}

No período de janeiro de 1980 a dezembro de 1988 , foram operados 128 pacientes por dissecções aórticas proximais, no Instituto do Coração do Hospital das Clínicas da Faculdade de Medicina da Universidade de São Paulo. Destes, em 66 a cirurgia foi realizada na fase aguda (até duas semanas do episódio inicial) Dos 62 doentes operados por dissecçōes crônicas, 44 (71\%) apresentavam insuficiência aórtica.

Analisando esse grupo de 44 pacientes, notamos que 35 eram do sexo masculino e 36 da raça branca. A idade variou de 23 a 66 anos, com média de 43 .

Em relação às etiologias mais freqüentes da dissecção, 26 (59\%) doentes eram hipertensos e sete $(16 \%)$ apresentavam síndrome de Marfan. Os sintomas mais comuns foram dor em $36(82 \%)$ e insuficência cardíaca em 32 (73\%). A insuficiência valvar era severa em 17 casos, moderada em 22 e leve nos cinco restantes.

A radiografia de tórax mostrou aumento da aorta em 37 ( $84 \%$ ) doentes e aumento da área cardíaca em $32(73 \%)$. O ecocardiograma préoperatório foi realizado em 30 pacientes, revelando insuficiência aórtica em todos. A cinecoronariografia realizada nos 44 casos mostrou insuficiência aórtica em todos, revelou orifício de entrada em 28 (64\%) e lesōes coronárias associadas em dois doentes.

Em $70 \%$ dos casos a dissecção era do tipo I. Em cinco pacientes, o ecocardiograma intra-operatório auxiliou na identificaçāo dinâmica da insuficiência valvar, que foi causada por um dos seguintes mecanismos: ectasia ânulo-aórtica, degeneraçāo valvar e desabamento das válvulas com alargamento do anel. Nos dois primeiros mecanismos optouse pela substituiçăo valvar, enquanto que no terceiro conservou-se a valva aórtica.

Em 41 (93\%) pacientes a aorta proximal foi substituída e nos três restantes realizou-se aortoplastia. Em $48 \%$ dos casos foi possível a preservaçāo valvar através da suspensāo da valva aórtica. Nos 23 doentes em que foi realizada a substituição valvar, a técnica de Bentall e de Bono foi utilizada em 16. Em seis pacientes foram associados outros procedimentos cirúrgicos. Em todos os pacientes operados, de 1986 em diante, foi utilizada cola biológica. A obliteraçāo da falsa luz através de sutura de parede da aorta entre duas tiras de feltro de Teflon antes da colocação do tubo de Dacron foi usada em todos os doentes.

Foi realizada curva atuaria! de sobrevida, para os pacientes que tiveram a valva aórtica substituída e para os que foram submetidos a plástica valvar.

Dezenove doentes foram avaliados tardiamente, através de um ou mais dos seguintes métodos: ecocardiograma, angiografia digital, angiografia convencional ou tomografia computadorizada do tórax.

A mortalidade imediata incluiu todos os pacientes que morreram durante a operaçāo, durante hospitalizaçāo ou nos primeiros 30 dias de pósoperatório.

\section{RESULTADOS}

Cinco $(11 \%)$ pacientes apresentaram mortalidade imediata e as causas do óbito foram estabelecidas em todos os pacientes através de necrópsia, sendo três por baixo débito, um por sangramento e um por complicaçāo neurológica. Dois $(4 \%)$ pacientes apresentaram morte súbita tardiamente. O seguimento dos 37 pacientes sobreviventes variou de dois a 108 meses, com média de $18 ; 78 \%$ estavam em classe funcional l e os demais em classe II. Dos dezenove doentes que foram reestudados por ecocardiograma, angiografia digital, angiografia convencional, ou tomografria, apenas dois pacientes em que se preservou a valva apresentaram insuficiência aórtica discreta. Nos pacientes com substituiçāo valvar, foi necessária a reoperaçāo em três que haviam recebido válvula biológica; nestes, foi utilizada, na reintervençāo, a técnica de Bentall e De Bono com protése de disco. Um doente, submetido inicialmente a aortoplastia e plástica valvar, apresentou redissecção e insuficiência aórtica após 60 meses, sendo reoperado pela técnica de Bentall.

Em estudo com curva atuarial, notamos que os pacientes submetidos a plástica valvar tiveram maior sobrevida do que aqueles em que a valva foi substituída (Gráfico 1).

\section{DISCUSSĀO}

As disssecçōes aórticas constituem afecçōes de gravidade variável segundo a localizaçāo e a duraçāo da doença. Segundo este último critério, ela tem sido classificada em aguda e crônica. A aguda corresponde ao período de até duas semanas, desde episódio inicial ${ }^{14}$.

Em relaçāo à localizaçāo, DE BAKEY et alii classificaram em tipo I, II e III, mas, atualmente, a 
PÊGo-FeRnANDES, P. M.; STOLF, N. A. G.; FONTES, R. D.; VERGINELLI, G.; JATENE, A. D. - Cirurgia das dissecçōes crônicas da aorta ascendente com insuficiência valvar. Rev. Bras. Cir. Cardiovasc., 5(3): 149-153, 1990.

GRÁFICO 1

PLÁSTICA VALVAR E SUBSTITUIÇÃO VALVAR CURVA ATUARIAL DE SOBREVIDA

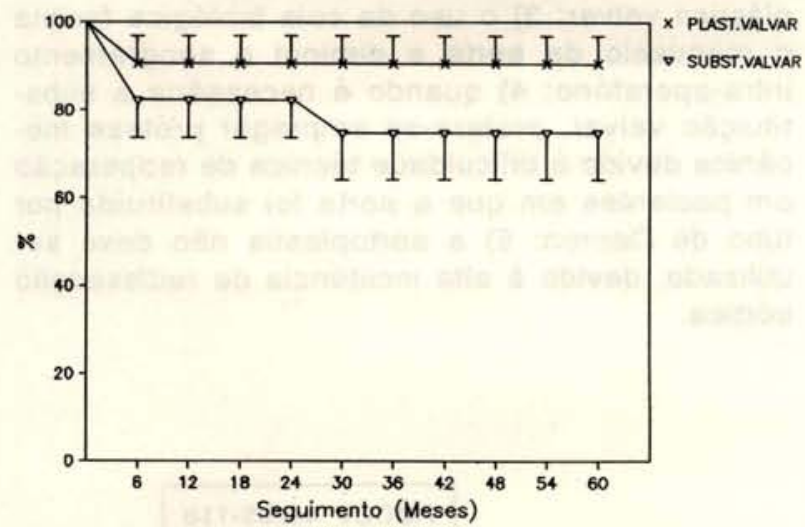

Os dados da curva atuarial foram submetidos a análise estatística através do método de contigência pelo teste do Qui-quadrado e os resultados nảo apresetaram diferença significante, provavelmente pelo número reduzido de casos. As barras do gráfico representam o erro padrão.

classificação mais aceita é a do grupo de Stanford ${ }^{7}$ que, do ponto de vista prognóstico e terapêutico, agrupou os tipos I e II no tipo A e o tipo III no tipo B. O tipo A é a dissecçāo que acomete a aorta ascendente e o tipo B aquela que se inicia a partir da aorta descedente.

Em relaçāo à terapêutica, aponta-se com uniformidade o tratamento cirúrgico como a conduta de escolha para as dissecçōes aórticas na fase crônica $3,5,9,10,13,18,20,22,23$, enquanto que essa posição é defendida mais freqüentemente apenas para as dissecçōes da aorta proximal na fase aguda ${ }^{8-10,18 .}$ 21, 25, 26 .

A correçāo cirúrgica das dissecçōes crônicas da aorta ascendente apresenta índices de mortalidade hospitalar que variam entre 4 e $20 \% 3,9,10.18$ ${ }^{20}$, sendo relatada uma sobrevida média de $75 \%$ dos pacientes operados ao final de cinco anos ${ }^{3,13,18}$ e de $56 \%$ aos 10 anos de seguimento ${ }^{13}$. O nosso resultado global no tratamento das dissecçōes aórticas crônicas proximais é de $9 \%{ }^{19}$, tendo-se realizado a ressecção do local de rotura primária da camada íntima da aorta em $93 \%$ desses pacientes 19. Destaca-se, na literatura, que a permanência do orifício de entrada das dissecçōes nāo tem influenciado os resultados imediatos de tratamento cirúrgico ${ }^{17}$, embora seja um fator de risco importante na evoluçāo tardia dos pacientes ${ }^{13}$.

Os melhores resultados na terapêutica cirúrgica no tratamento das dissecçōes crônicas da aorta proximal devem-se, principalmente, à baixa incidência de complicaçōes secundárias à dissecçāo, nessa fase ${ }^{14}$. 19. Temos utilizado a cola biológica des- crita por GUILMET et alii ${ }^{12}$ para todos os casos de dissecçāo aórtica, agudos ou crônicos. A insuficiência aórtica, que é observada com freqüência na fase crônica, nāo tem elevado o risco cirúrgico, nesses pacientes, independentemente da técnica empregada para sua correçāo $9,10,14,18,19$. A incidência de insuficiência aórtica, na nossa casuística, é de $71 \%$ nas dissecçōes crônicas proximais, sendo de $55 \%$ nas dissecçōes agudas ${ }^{19}$. JEX et alii ${ }^{14}$ relataram uma incidência global de $58 \%$ de insuficiência aórtica nas dissecçōes aórticas e de $72 \%$ nas dissecçōes crônicas, semelhantes à de outros autores ${ }^{18}$. JEX et alii ${ }^{14}$ atribuíram a maior incidência da insuficiência aórtica nas dissecçōes crônicas à seleçāo natural e ao fato de que o diagnóstico das dissecçōes crônicas é mais fácil de ser feito nos pacientes com manifestaçōes clínicas de insuficiência valvar.

Há controvérsias em relaçāo à melhor terapêutica da insuficiência aórtica nas dissecçōes aórticas. $\mathrm{Na}$ fase aguda, nosso grupo preservou a valva aórtica em $96 \%$ dos casos com insuficiência aórtica ${ }^{19}$, MILLER et alii ${ }^{17}$, em $82 \%$, outros ${ }^{14}$ em $40 \%$, enquanto há outros ${ }^{3,16}$ que preferem a utilização de tubo valvulado e reimplante de coronárias no tratamento dos aneurismas e dissecçōes aórticas agudas ou crônicas. $\mathrm{Na}$ fase crônica, a insuficiência aórtica nāo tem elevado o risco cirúrgico independentemente da técnica empregada para sua correçăo 3, 9, 10, 14, 16, 18, 19. A substituição da valva aórtica tem sido mais utilizada (em até $100 \%$ dos casos) no tratamento da insuficiência aórtica secundária à dissecçāo da aorta ascendente 9.10,14, 21,23, do que os procedimentos conservadores ${ }^{10,17-19}$. No início de nossa experiência ${ }^{23}$ substituímos a valva aórtica com maior freqüência (em $78,3 \%$ dos casos). $\mathrm{Na}$ medida em que adquirimos confiança na plástica valvar ${ }^{19}$, passamos a tentar a conservaçāo dessa valva nas dissecçōes aórticas crônicas, com excessão dos casos em que haja ectasia ânuloaórtica ou síndrome de Marfan, ou acometimento primário valvar. Nos casos de ectasia ânulo-aórtica ou de Marfan, temos utilizado o tubo valvulado com reimplante de coronárias, como descrito por Bentall e De Bono, assim como outros autores 5, 6, 11, 15-17. A técnica descrita por CABROL et alii ${ }^{2}$ - alternativa ao reimplante direto das coronárias ao tubo de Dacron-valvulado, com a utilização de um pequeno tubo colocado transversalmente ao tubo de Dacron e suturado aos óstios coronários - também tem sido utilizada na literatura, com bons resultados ${ }^{3,4}$. Mais recentemente, mesmo os autores ${ }^{14,20}$ que não utilizam muito a plástica valvar nas dissecçōes aórticas crônicas com insuficiência aórtica, mostram uma predisposição para empregá-la com maior freqüência. Assim como nós, eles notaram complicaçōes devido ao uso de substituiçāo valvar. JEX et alii ${ }^{14}$ mostraram melhor resultado tardio nos casos em que preservaram a valva, assim como observado 
PÊGo-FERNANDES, P. M.; STOLF, N. A. G.; FONTES, R. D.; VERGINELLI, G.; JATENE, A. D. - Cirurgia das dissecçōes crônicas da aorta ascendente com insuficiência valvar. Rev. Bras. Cir. Cardiovasc., 5(3): 149-153, 1990.

neste trabalho. Em relação à aortoplastia estes mesmos autores relataram $31 \%$ de redissecção aórtica com o uso dessa técnica. Nós a utilizamos em apenas três pacientes, e um deles apresentou recorrência (33\%), necessitando de reoperaçāo e utilizaçăo da técnica de Bentall e De Bono.

Podemos concluir que: 1) a suspençāo valvar é uma técnica satisfatória em pacientes com dissecçōes crônicas da aorta, com baixa mortalidade e menor incidência de complicaçōes a médio prazo do que a substituiçāo valvar; 2) a identificaçāo do mecanismo da insuficiência valvar é fundamental e o ecocardiograma intra-operatório pode nos auxiliar nessa decisāo e na avaliaçāo imediata da plástica valvar; 3) o uso da cola biológica facilita - manuseio da aorta e diminui o sangramento intra-operatório; 4) quando é necessária a substituiçāo valvar, prefere-se empregar prótese mecânica devido à dificuldade técnica de reoperaçāo em pacientes em que a aorta foi substituída por tubo de Dacron; 5) a aortoplastia nāo deve ser utilizada, devido à alta incidência de redissecçāo aórtica.

\section{RBCCV 44205-118}

PÊGO-FERNANDES, P. M.; STOLF, N. A. G.; FONTES, R. D.; VERGINELLI, G.; JATENE, A. D. - Surgery of chronic aortic dissection with aortic insufficiency. Rev. Bras. Cir. Cardiovasc., 5(3): 149-153, 1990.

ABSTRACT: In the period of January 1980 to December 1988, 44 patients with chronic aortic dissections and aortic insufficiency were operated on. This group of patients was analized in order to evaluate the evolution of those in which the aortic valve was preserved compared to the group of patients submitted to valvular replacement. The overall preoperative characteristics of these two groups were similar. Valvular replacement was the elected procedure in cases of valvular degeneration or of aortic annular ectasia. In cases of cusp prolapse with enlarged annulus a plastic procedure was used; in $48 \%$ of the cases it was possible to preserve the valve. In the 23 patients submitted to valve replacement, the Bentall and De Bono technique was utilized. In six patientes other surgical procedures were associated. Biological adhesives were utilized in every patient operated on from 1986 on. In 41 patients (93\%) the proximal aorta was substituted and in the remaining three an aortoplasty was performed. Five patients (11\%) had hospitalar deaths, three due to low-output syndrome, one due to bleeding and one on account of neurological complications. Late death occurred in two patients $(4 \%)$. The follow-up of the 37 surviving patients varied from two to 108 months (mean: 18 months); of these, $78 \%$ were in fuctional class 1 , and the others in class II. Two patients that had their aortic valve preserved presented mild aortic insufficiency. Three patients with bioprosthesis were reoperated on due to dysfunction. One patient submitted to aortoplasdty and an aortic valve plastic procedure presenting redissection and aortic insufficiency after 60 months, was reoperated on using the Bentall technique. In the actuarial curve analysis, patients submitted to valvuloplastic procedures had longer survival rates than the valve replacement patients. It is possible to conclude that: 1) valvular resuspension is a satisfactory technique in patients with chronic dissection of the aorta, with low mortality and less complications than valvular replacement; 2) identification of the mechanism producing the valvular insufficiency is fundamental for the choice of the surgical procedure; 3) the use of biological adhesives render easy the handling of the aorta and lessen the intraoperative bleeding; 4) when valvular replacement is indicated, mechanical prostheses are preferred, since reoperations are more difficult in these patients; 5 ) aortoplasties are avoided due to the high incidence of aortic redissection.

DESCRIPTORS: aortic dissection, surgery; heart valves, aortic, surgery.

\section{REFERÊNCIAS BIBLIOGRÁFICAS}

1 BENTAL, H. H. \& De BONO, A. - A technique for complete replacement of the ascending aorta. Thorax, 23: $338-339,1968$.

2 CABROL, C.; GANDJBAKHCH, I.; CHAM, B. - Anévrismes de l'aorte ascendante: replacemet total avec réimplantation des artères coronaires. Nouv. Presse Med., 7: 363-365, 1978.
3 CABROL, C.; PAVIE, A.; MESNILDREY, P.; GRANDJBAKHCH, I.; LAUGHLIN, K; BORS, V.; CORCOS, $\mathrm{T}$ - Long-term results with total replacement of ascending aorta and reimplantation of the coronary arteries. J. Thorac. Cardiovasc. Surg., 91: 17-25, 1986.

4 COSELI, J. S. \& CRAWFORD, E. S. - Composite valvegraft replacement of aortic root using separate Dacron tube coronary artery reattachment. Ann. Thorac. Surg. 47: 558-565, 1989. 
PÊGo-FERnANDES, P. M.; STOLF, N. A. G.; FONTES, R. D.; VERGINELLI, G.; JATENE, A. D. - Cirurgia das dissecçöes crônicas da aorta ascendente com insuficiência valvar. Rev. Bras. Cir. Cardiovasc., 5(3): 149-153, 1990.

5 CRAWFORD, E. S.; CRAWFORD, J. L.; STOWE, C. L.; SAFI, H. J. - Total aortic replacement for chronic aortic dissection occurring in patients with or without Marfan syndrome. Ann. Surg., 199: 358$362,1984$.

CULLIFORD, A. T.; AYVALIOTIS, B.; SHEMIN, R.; COLVIN, S.B.; ISOM, O.W.; SPENCER, F. C.; - Aneurysms of the ascending aorta and transverse arch: surgical experience in 80 patients. J. Thorac. Cardiovasc. Surg., 83: 701-710, 1982.

DAILY, P. O.; TRUEBLOOD, H. W.; STINSON, E. B.; WUERFLEIN, R. D.; SHUMWAY, N. E. - Management of acute aortic dissections. Ann. Thorac. Surg., 10: 237-247, 1970.

DeBAKEY, M. E.; HENLY, W. A.; COOLEY, D. A.; MORRIS, G. C.; CRAWFORD, E. S.; BEALL, A. C. - Surgical management of dissecting aneurysms of the aorta. J. Thorac. Cardiovasc. Surg., 49: 130$149,1965$.

9 DeBACKEY, M. E.; McCOLLUM, C. H.; CRAWFORD, E. J.; MORRIS, G. C.; HOWELL, J.; NOON, G. P.; LAURIE, G. -Dissection and dissecting aneurysms of the aorta: twenty-year follow-up of five hundred twenty-seven patients treated surgically. Surgery, 92: 1118-1134. 1982.

DOROGHAZI, R. M.; SLATER, E. E.; DeSANCTIS, R. W.; BUCKLEY, M. J.; AUSTEN, W. G.; ROSENTHAL, S. - Long-term survival of pacients with treated aortic dissection. J. Am. Coll. Cardiol., 3: 1026-1034, 1984.

11 GOTT, V. L.; PYERITZ, R. E.; Mc GOVERN Jr., G. J; CAMERON, D. E.; Mc KUSICK, V.A. - Surgical treatment of aneurysms of the ascending aorta in the Marfan syndrome: results of composite graft repair in 50 patients. N. Engl. J. Med., 314: 1070-1074, 1986.

GUILMET, D.; BACHET, J.; GOUDOT, B.; LAURIAN, C.; GIGOU, F.; BICAL, O.; BARBAGELATTA, M. Use of biological glue in acute aortic dissection: preliminary clinical results with a new surgical technique. J. Thorac. Cardiovasc. Surg., 77: 516-521, 1979.

HAVERICH, A.; MILLER, D. C.; SCOTT, W. C.; MITCHELL, R. S.; OYER, P. E.; STINSON, E. B.; SHUMWAY, N. E. - Acute and chronic aortic dissections: determinants of long-term outcome for operative survivors. Circulation, 72 (Pt. 2): 22-34, 1985.

14 JEX, R. K.; SCHAFF, H. V ; PIEHLER, J. M.; ORSZULAK, T. A.; PUGA, F. J.; KING, M.; DANIELSON, G. K.; PLUTH, J. R. - Repair of ascending aortic dissection: influence of associated aortic valve insufficiency on early and late results. J. Thorac. Cardiovasc. Surg., 93: 375-384, 1987.

15 Mc DONALD, G. R.; SCHAFF, H. V.; PYERITZ, R. E.; McKUSICK, V. A.; GOTT, V. L. - Surgical management of patients with the Marfan's syndrome and dilatation of the ascending aorta. J. Thorac. Cardiovasc. Surg., 81: 180-186, 1981.

MARVASTI , M. A.; PARKER Jr., F. B.; RANDALL, P. A.; WITWER, G. A. - Composite graft replacement of the ascending aorta and aortic valve: late followup with intra-arterial digital subtraction angiography. J. Thorac. Cardiovasc. Surg, 95: 924-928, 1988.

17 MILLER, D. C.; MITCHELL, R. S.; OYER, P. E.; STINSON, E. B.; JAMIESON, S. W.; SHUMWAY, N. E.. Independent determinants of operative mortality for patients with aortic dissections. Circulation, 70: 153-164, 1984. S. J.; REITS, B. A.; GRIEPP, R. B.; SHUMWAY, N. E. - Operative treatment of aortic dissectiones: experience with 125 patientes over a sixteen-year period. J. Thorac. Cardiovasc. Surg., 78: 365-382, 1979.

MOREIRA,L. F. P.; STOLF, N. A. G.: VIANNA, C. B.; PÊGO-FERNANDES, P. M.; BARRETO, A. C. P. VERGINELLI, G.; JATENE, A. D. - Fatores de risco na cirurgia de dissecçāo da aorta ascendente e arco aórtico Rev. Bras. Cir. Cardiovasc., 2: 121:128, 1987.

PRESSLER, V. \& Mc NAMARA, J. J.. - Aneurysm of thoracic aorta: review of the 260 cases. J. Thorac. Cardiovasc. Surg., 89: 50-54, 1985.

SAMPAIO, F. A. F.; VILA, J. H.; MELO, R. F. A; BITTENCOURT, D.; SOUZA, M. C.; GALIANO, N.; MACRUZ, R.; ZERBINI, E. J. - Dissecção aguda da aorta: seguimento imediato e tardio de pacientes submetidos a tratamento clínico e cirúrgico. Arq. Bras. Cardiol., 45: 385-387, 1985.

STOLF, N. A. G.; PÊGO-FERNANDES, P. M., BITTENCOURT, D.; BARRETO, A. C. P.; VERGINELLI, G.; PILEGGI, F.; ZERBINI, E. J. - Aneurismas e dissecçōes da aorta torácica: tratamento cirúrgico. Arq. Bras. Cardiol., 41: 221-226, 1983.

STOLF, N. A. G.; PÊGO-FERNANDES, P. M., COSTA, R.; BARRETO, A. C. P.; PILEGGI, F.; VERGINELLI, G. - Tratamento cirúrgico das dissecçōes crônicas da aorta torácica. Rev. Hosp. Clínicas Fac. Med. (S. Paulo), 38: 3-7, 1983.

24 THOMAS Jr., C. S.; ALFORD Jr., W. C.; BURRUS, G. R.; FRIST, R. A.; STONEY, W. S. - The effectiveness of surgical treatment of acute aortic dissection. Ann. Thorac. Surg., 26: 42-49, 1978.

25 WHEAT Jr., M. W.; HARRIS, P. D.; MALM, M. R.; KAISER, G.; BOWMAN Jr., F. O.; PALMER, R. F. - Acute dissecting aneurysms of the aorta. J. Thorac. Cardiovasc. Surg., 58: 344-381, 1969.

26 WOLFE, W. G. \& MORAN, J. F. - The evolution of medical and surgical management of acute aortic dissection. Circulation, 56: 503-505, 1977. 